Communications in Physics, Vol.20, No. 2 (2010), pp. 97-111

\title{
TENSOR TWO-LOOP SELF-ENERGY INTEGRALS
}

\author{
PHAN HONG KHIEM AND DO HOANG SON \\ Ho Chi Minh City University of Science
}

\begin{abstract}
Based on the technique for evaluating tensor two-loop self-energy integrals in parallel and orthogonal spaces [1], we present a new UV subtraction procedure for a subset of integrals which contains overall UV-divergence. The numerical and analytical results of the sun-rise integral are discussed in comparison with other methods.
\end{abstract}

\section{INTRODUCTION}

High precision measurements at LHC and future colliders like ILC require an equivalent accuracy in theoretical predictions. To check the validity of the Standard Model, complex calculations dealing with huge numbers of Feynman graphs up to two-loop level within the perturbative theory are necessary. In the last few years, there have had many proposed techniques to calculate massive two-loop selfenergy tensor integrals. It is clear that, up to now, there are no perfect solutions and perfect techniques for the problem. Indeed, this is a very hard and active topic of multi-loop and multi-leg problem, a branch of computational high energy physics. A complete review can be found in $[2,3]$.

In this article, we present a technique to calculate two-loop selfenergy tensor integrals with arbitrary masses in parallel/orthogonal spaces. Kreimer [1] has provided a general and constructive method to express massive two-loop integrals in terms of finite integrals suitable to numerical evaluations plus a set of products of one-loop integrals contains the UV singular part. The separation of the UV-divergent part from its counter UV-finite part can be obtained by a subtraction procedure in which subtraction terms are provided explicitly. From the computational point of view, the method provides a simple recipe to develop a computer program for calculating arbitrary tensor massive two-loop integrals.

However, detailed studies on the structure of tensor two-loop self-energy integrals which contain overall UV-divergences $[4,5]$ show that in order to isolate the overall UVdivergence, the method needs to be revised to avoid the intervention of spurious IRdivergences from the subtraction terms.

In this paper, we present in detail a new subtraction procedure which completes the Kreimer's method and is applicable for a subset of degenerate tensor two-loop self-energy integrals. The new subtraction procedure is then implemented into the XLOOPS-GiNaC program package [6] which provided an independent and a strong tool to the physics community. To test the accuracy and the performance of the program and of the method we present results that using XLOOPS-GiNaC to evaluate sunrise two-loop integrals in comparison with various results of other authors when it is possible. 
Even though this paper is dedicated to the two-loop self-energy integrals, the technique is also applicable to an arbitrary two-loop tensor integral [7].

\section{TENSOR TWO-LOOP SELF-ENERGY INTEGRALS AND THE REDUCTION}

A general tensor two-loop integral has the following representation

$$
\begin{aligned}
T_{t_{1} \ldots t_{n} ; \ldots t_{n}+n_{m} ; \ldots n_{n_{l}+n_{m}+n_{k}}}^{(2) \mu_{1} \ldots \mu_{p}, \nu_{1} \ldots \nu_{r}}=\int d^{D} l & \int d^{D} k \frac{l^{\mu_{1}} \ldots l^{\mu_{p}}}{\prod_{s=1}^{n_{l}}\left[\left(l+q_{s}\right)^{2}-m_{s}^{2}+i \rho\right]^{t_{s}}} \\
& \times \frac{1}{\prod_{s=n_{l}+1}^{n_{l}+n_{m}}\left[\left(l+k+q_{s}\right)^{2}-m_{s}^{2}+i \rho\right]^{t_{s}}} \\
& \times \frac{k^{\nu_{1}} \ldots k^{\nu_{r}}}{n_{l}+n_{m}+n_{k}}\left[\left(k+q_{s}\right)^{2}-m_{s}^{2}+i \rho\right]^{t_{s}}
\end{aligned}
$$

where $n_{l}, n_{k}, n_{m}$ are the numbers of propagators which carry the loop momenta $l, k$ or $l+k$ respectively. $q_{s}$ and $m_{s}$ are external momenta and masses of particles involved.

It is believed that, beyond the one-loop level, the result of a general massive integral may not be expressible in terms of polylogarithms $[8,9]$. To integrate an integral such as the one in Eq. (1), one introduces a set of proper subtraction terms to separate the singular parts (UV or IR singularities) into analytically solvable integrals and finite integrals which then can be integrated numerically.

For two-loop self-energy integrals, there are four non-trivial topologies shown in Fig.(1), which are not able to be reduced to another topologies or to products of one-loop ones

Different to the Passarino-Veltman's reduction technique, we are going to evaluate Feynman integrals which are associated with diagrams in Fig.(1) by working in a specific frame of reference where the momentum of the incoming particle has only one none vanishing component ${ }^{1}$. In such a frame of reference, for example a space-like particle with $q^{2}>0$, one can parameterize

$$
q^{\mu}=\left(q_{0}, \overrightarrow{0}\right)
$$

with $\overrightarrow{0}$ is the null-vector in $D-1$ dimension space.

\footnotetext{
${ }^{1}$ Note that, we can always find down such frame of references if the external momentum $q^{\mu}$ is spacelike or time-like. If the external momenta is light-like, $q^{2}=0$, the integral cannot be directly calculated using parallel and orthogonal technique. For this special case, the integral can be evaluated by reducing to scalar integrals [10] that in turn can be evaluated using various techniques such as in [11-14].
} 


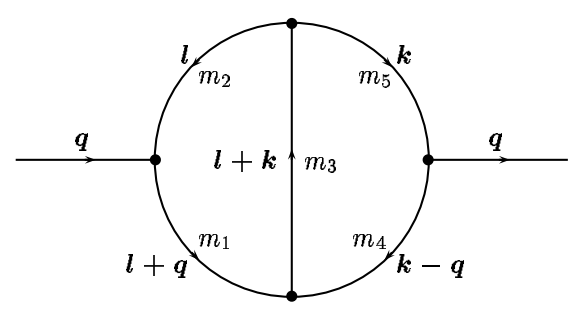

(a)

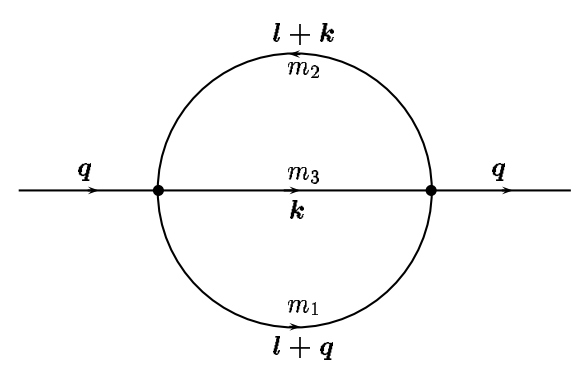

(c)

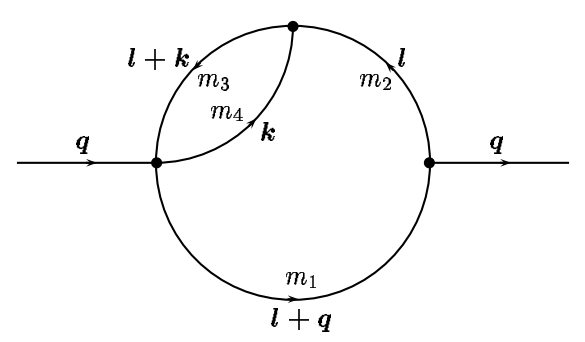

(b)

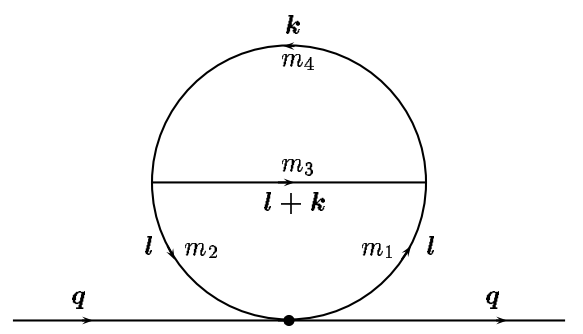

(d)

Fig. 1. The non-trivial two-loop self-energy topologies.

In this frame of reference, the components of loop momenta in the parallel and orthogonal spaces spanned by the external momentum $q^{\mu}$ can be defined by

$$
\begin{aligned}
l_{0} & =\frac{l \cdot q}{\sqrt{q^{2}}}, \quad k_{0}=\frac{k \cdot q}{\sqrt{q^{2}}}, \\
l_{\perp}^{\mu} & =l^{\mu}-\frac{l \cdot q}{q^{2}} q^{\mu}=\left(0, \vec{l}_{\perp}\right) \\
k_{\perp}^{\mu} & =k^{\mu}-\frac{k \cdot q}{q^{2}} q^{\mu}=\left(0, \vec{k}_{\perp}\right)
\end{aligned}
$$

with $\vec{l}_{\perp}$ and $\vec{k}_{\perp}$ are $D-1$ dimension vectors in the perpendicular sub-space to $q^{\mu} .{ }^{2}$

\footnotetext{
${ }^{2}$ For the time-like cases where $q^{2}<0$ we can process in a similar manner after rearranging the indices.
} 
In terms of the new variables, the integrals which are associated with topologies in Fig.(1) can be rewritten respectively in the form

$$
\begin{gathered}
T^{a}=\int d^{D} l \int d^{D} k \frac{l_{0}^{p_{0}} l_{\perp}^{p_{\perp}} k_{0}^{r_{0}} k_{\perp}^{r_{\perp}} z^{p_{z}}}{\left[(l+q)^{2}-m_{1}^{2}\right]^{t_{1}}\left[l^{2}-m_{2}^{2}\right]^{t_{2}}} \times \\
\times \frac{1}{\left[(l+k)^{2}-m_{3}^{2}\right]^{t_{3}}\left[(k-q)^{2}-m_{4}^{2}\right]^{t_{4}}\left[k^{2}-m_{5}^{2}\right]^{t_{5}}}, \\
T^{(b)}=\int d^{D} l \int d^{D} k \frac{l_{0}^{p_{0}} l_{\perp}^{p_{\perp}} k_{0}^{r_{0}} k_{\perp}^{r_{\perp}} z^{p_{z}}}{\left[(l+q)^{2}-m_{1}^{2}\right]^{t_{1}}\left[l^{2}-m_{2}^{2}\right]^{t_{2}}\left[(l+k)^{2}-m_{3}^{2}\right]^{t_{3}}\left[k^{2}-m_{4}^{2}\right]^{t_{4}}}, \\
T^{(c)=} \int d^{D} l \int d^{D} k \frac{l_{0}^{p_{0}} l_{\perp}^{p_{\perp}} k_{0}^{r_{0}} k_{\perp}^{r_{\perp}} z^{p_{z}}}{\left[(l+q)^{2}-m_{1}^{2}\right]^{t_{1}}\left[(l+k)^{2}-m_{2}^{2}\right]^{t_{2}}\left[k^{2}-m_{3}^{2}\right]^{t_{3}}}, \\
T^{(d)=} \int d^{D} l \int d^{D} k \frac{l_{0}^{p_{0}} l_{\perp}^{p_{\perp}} k_{0}^{r_{0}} k_{\perp}^{r_{\perp}} z^{p_{z}}}{\left[l^{2}-m_{1}^{2}\right]^{t_{1}}\left[l^{2}-m_{2}^{2}\right]^{t_{2}}} \frac{1}{\left[(l+k)^{2}-m_{3}^{2}\right]^{t_{3}}\left[k^{2}-m_{4}^{2}\right]^{t_{4}}}
\end{gathered}
$$

where $z$ is the cosine of the angle between $\vec{l}_{\perp}$ and $\vec{k}_{\perp}$. The variables $p_{0}, p_{\perp}, r_{0}, r_{\perp}, p_{z}$ are non-negative integers and $t_{i}$ are positive integers. Detailed discussions on the transformation of the standard tensor integrals, as in Eq. (1), to a parallel and orthogonal space representation can be found in $[4,15]$. Here and to the rest of the paper, we will call the integrals in Eq. (4) to Eq. (7) tensor integrals in the parallel and orthogonal representation or simply tensor integrals if there is any confuse.

Working in this representation has a big advantage in performing tensor reduction $[4,16-18]$. The other advantage is that we can find out a two-dimensional representation for the finite part of the integral directly by using the residuum theorem without performing the Feynman parametrisation.

It is worth to note here that the non-negative integers $p_{0}, p_{\perp}, r_{0}, r_{\perp}, p_{z}$ are not Lorentz indices. They are exponents of the real variables $l_{0}, l_{\perp}, k_{0}, k_{\perp}$ and $z$ respectively. In general, $p_{\perp}$ and $r_{\perp}$ can be odd or even due to the fact that the denominators of the integrands in Eq. (4) to Eq. (7) are not symmetric either in $l_{\perp}$ or in $k_{\perp}$ separately. However,

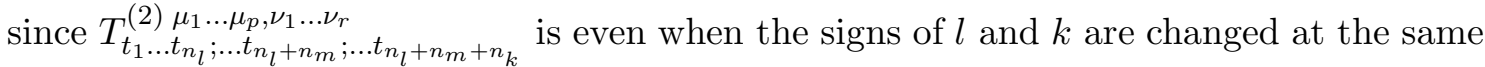
time, thus one has other constraints:

- The integrals with odd $\left(p_{\perp}+r_{\perp}\right)$ vanish.

- $p_{z}$ must be smaller than or equal to $\min \left\{p_{\perp}, r_{\perp}\right\}$.

- Both $\left(p_{\perp}-p_{z}\right)$ and $\left(r_{\perp}-p_{z}\right)$ are even.

In general, the integrals in Eq. (4) to Eq. (7) can be degenerate. However, by a simple reduction, we will prove that all the integrals will be reduced to a sum of none-degenerate integrals and degenerate integrals of the form $T^{(c)}$. It means that the degenerate problem stays only in the sunrise integral, the Eq. (6).

To have a closer look at this reduction, let us consider the reduction of the integral in Eq. (4). 


\section{II.1. Tensor reduction}

We first rewrite the integral in Eq. (4) in the following form

$$
T^{(a)}\left(q^{2}\right)=\int d^{D} l \int d^{D} k \frac{l_{0}^{p_{0}} l_{\perp}^{p_{\perp}} k_{0}^{r_{0}} k_{\perp}^{r_{\perp}} z^{p_{z}}}{P_{1}^{t_{1}}(l+q) P_{2}^{t_{2}}(l) P_{3}^{t_{3}}(l+k) P_{4}^{t_{4}}(k-q) P_{5}^{t_{5}}(k)}
$$

with

$$
\begin{aligned}
P_{1}(l+q) & =(l+q)^{2}-m_{1}^{2}+i \rho, \\
P_{2}(l) & =l^{2}-m_{2}^{2}+i \rho, \\
P_{3}(l+k) & =(l+k)^{2}-m_{3}^{2}+i \rho, \\
P_{4}(k-q) & =(k-q)^{2}-m_{4}^{2}+i \rho, \\
P_{5}(k) & =k^{2}-m_{5}^{2}+i \rho .
\end{aligned}
$$

We can always remove the variable $z$ in the numerator by inserting

$$
l_{\perp} k_{\perp} z=\frac{1}{2}\left[-P_{3}+\left(l_{0}+k_{0}\right)^{2}-l_{\perp}^{2}-k_{\perp}^{2}-m_{3}^{2}-i \rho\right]
$$

into the numerator of the integral in Eq. (8). After expanding recursively, one obtains only three basics integrals of the form

$$
\begin{aligned}
J_{0} & =\int d^{D} l \int d^{D} k \frac{1}{P_{1}^{t_{1}}(l+q) P_{2}^{t_{2}}(l) P_{3}^{t_{3}}(l+k) P_{4}^{t_{4}}(k-q) P_{5}^{t_{5}}(k)} \\
J_{1} & =\int d^{D} l \int d^{D} k \frac{k_{0}^{r_{0}}}{P_{1}^{t_{1}}(l+q) P_{2}^{t_{2}}(l) P_{3}^{t_{3}}(l+k) P_{4}^{t_{4}}(k)} \\
J_{2} & =\int d^{D} l \int d^{D} k \frac{l_{0}^{p_{0}} k_{0}^{r_{0}}}{P_{1}^{t_{1}}(l+q) P_{2}^{t_{2}}(l+k) P_{3}^{t_{3}}(k)}
\end{aligned}
$$

The integral $J_{0}$ is finite so that one can perform a numerical integration directly. A two-fold representation for the numerical integration is discussed in [16]. The topology of two-point diagrams with three propagators is called sunrise topology. Its associated integrals, such as $T^{(c)}$ or $J_{2}$, are called sunrise integrals. By the same procedure, the two other tensor integrals which are associated with topologies (b) and (c) can also be reduced to expressions containing $J_{1}, J_{2}$ and products of one-loop functions.

\section{II.2. Power counting and degenerate integrals}

In order to construct the subtraction terms for the integral $J_{1}, J_{2}$, let us define some notations.

Let $w$ denotes the overall degree of UV-divergence and $w_{l}, w_{k}, w_{l k}$ are degrees of $\mathrm{UV}$-sub-divergences. By power counting, for $J_{1}$ we define

$$
\begin{aligned}
w_{l} & =2\left(t_{1}+t_{2}+t_{3}\right)-D>0, \\
w_{k} & =2\left(t_{3}+t_{4}\right)-D-r_{0}, \\
w_{l k} & =2\left(t_{1}+t_{2}+t_{4}\right)-D-r_{0} \\
w & =\frac{1}{2}\left(w_{l}+w_{k}+w_{l k}-D\right) .
\end{aligned}
$$


and for $J_{2}$

$$
\begin{aligned}
w_{l} & =2\left(t_{1}+t_{2}\right)-D-p_{0}, \\
w_{k} & =2\left(t_{2}+t_{3}\right)-D-r_{0}, \\
w_{l k} & =2\left(t_{1}+t_{3}\right)-D-p_{0}-r_{0} \\
w & =\frac{1}{2}\left(w_{l}+w_{k}+w_{l k}-D\right) .
\end{aligned}
$$

The condition for UV-finite of an integral is that all of its degrees of UV-divergence are positive. We call an UV-divergent integral degenerate if it is truly overall UV-divergent. By power counting, an integral is degenerate if

$$
w_{l}+w_{k}+w_{l k} \leq D .
$$

Otherwise, the integral is said non-degenerate. The method for evaluating a non-degenerate integral is presented in detailed in [1] which we will not review in this paper. From now on, we will consider only the degenerate integrals. It is clear that from the power counting condition, $J_{0}$ is finite and can then be integrated numerically in two-dimension space and was discussed in detailed in $[5,16]$.

In this paper, we consider only the case where $w_{l k}>0$. In this condition, $J_{1}$ is nondegenerate with any positive value of $r_{0}$ and the only degenerate integral is of the type $J_{2}$. The integral $J_{1}$ is also be integrated with a suitable subtraction terms as in $[4,5,16]$. To complete the calculation, we have to consider the case where $w_{l k} \leq 0$. However this problem will be discussed separately in another paper.

To close this section, we conclude that only integrals of type $J_{2}$ can be degenerate. This type of integrals is the main interest of this paper. In the next section, we start to construct the subtraction terms for the degenerate integral $J_{2}$ with a condition $w_{l k}>0$.

\section{II.3. The degenerate integral $J_{2}$ and its subtraction terms}

In this section, we consider the integral $J_{2}$ introduced in Eq. (13) which appeared as a result of the tensor reduction

$$
J_{2}=\int d^{D} l d^{D} k \mathcal{J}_{2}^{p_{0}, r_{0}} \quad(i=1,2,3)
$$

with the condition

$$
w_{l k}=2\left(t_{1}+t_{3}\right)-D-p_{0}-r_{0}>0 .
$$

Let us rewrite the integrand

$$
\mathcal{J}_{2\left\{t_{i}\right\}}^{p_{0}, r_{0}}=\frac{l_{0}^{p_{0}} k_{0}^{r_{0}}}{P_{1}^{t_{1}}(l+q) P_{2}^{t_{2}}(l+k) P_{3}^{t_{3}}(k)}
$$

where

$$
\begin{aligned}
P_{1}(l+q) & =(l+q)^{2}-m_{1}^{2}+i \rho, \\
P_{2}(l+k) & =(l+k)^{2}-m_{2}^{2}+i \rho, \\
P_{3}(k) & =k^{2}-m_{3}^{2}+i \rho .
\end{aligned}
$$

By power counting, if $w_{l} \geq 2 t_{2}$ or $w_{k} \geq 2 t_{2}$ the integral is non-degenerate and one can evaluate by the same procedure as described in [1]. 
We now suppose that $w_{l} \leq 0, w_{k} \leq 0$ and $w \leq 0^{3}$. In order to remove all divergences, one needs subtraction terms to remove the overall-, the $l$ - and the $k$-sub-divergences. The trick is to use a massive subtraction term to increase the degree of $l$-sub-divergence to $2 t_{2}$ and isolate the overall divergence in a scalar two-loop vacuum integral $T^{(d)}$ (see Eq. $(7)$ ) which is integrable [11-14]. The $k$-sub-divergence is still subtracted off by the massless subtraction term as in [1]. We define subtraction terms

$$
\begin{aligned}
L^{x_{l}} & :=\left(1-\frac{P_{1}^{t_{1}}}{\bar{P}_{1}^{t_{1}}}\right)^{x_{l}}=1+L_{0}^{x_{l}}, \\
K^{x_{k}} & :=\left(1-\frac{P_{2}^{t_{2}} P_{3} t_{3}}{\widetilde{P}_{2}^{t_{2}} \widetilde{P}_{3}^{t_{3}}}\right)^{x_{k}}=1+K_{0}^{x_{k}}
\end{aligned}
$$

where

$$
\begin{aligned}
\bar{P}_{1} & :=l^{2}-m_{1}^{2}+i \rho, \quad \widetilde{P}_{2}:=(l+k)^{2}, \quad \widetilde{P}_{3}:=k^{2}, \\
L_{0}^{x_{l}} & =\sum_{n=1}^{x_{l}}\left(\begin{array}{c}
x_{l} \\
n
\end{array}\right)(-1)^{n}\left(\frac{P_{1}^{t_{1}}}{\bar{P}_{1}^{t_{1}}}\right)^{n}, \\
K_{0}^{x_{k}} & =\sum_{n=1}^{x_{k}}\left(\begin{array}{c}
x_{k} \\
n
\end{array}\right)(-1)^{n}\left(\frac{P_{2}^{t_{2}} P_{3}^{t_{3}}}{\widetilde{P}_{2}^{t_{2}} \widetilde{P}_{3}^{t_{3}}}\right)^{n} .
\end{aligned}
$$

$x_{l}$ and $x_{k}$ are chosen such that

$$
x_{l}+w_{l}=2 t_{2}, \quad x_{k}+w_{k}=1 .
$$

In contrast to $K$, the subtraction term $L$ is constructed by a massive propagator $1 / \bar{P}_{1}$ that allows one to choose $x_{l}$ big enough to make the whole integral finite without introducing an IR-divergence. This is the main difference to [1]. Using subtraction terms $L$ and $K$, the integral can be rewritten as follows

$$
\begin{aligned}
J_{2}= & \int d^{4} l d^{4} k \mathcal{J}_{2\left\{t_{1}\right\}}^{p_{0}, r_{0}} L^{x_{l}} K^{x_{k}}-\int d^{D} l d^{D} k \mathcal{J}_{2\left\{t_{i}\right\}}^{p_{0}, r_{0}} L_{0}^{x_{l}} \\
& -\int d^{D} l d^{D} k \mathcal{J}_{2\left\{t_{i}\right\}}^{p_{0}, r_{0}} L_{0}^{x_{l}} K_{0}^{x_{k}}-\int d^{D} l d^{D} k \mathcal{J}_{2\left\{t_{i}\right\}}^{p_{0}, r_{0}} K_{0}^{x_{k}} .
\end{aligned}
$$

The first term on the right-hand side of Eq. (21) is finite; so it can be integrated numerically in $D=4$ dimensions. The last two terms are massless $k$-integrals which can be reduced to the integral

$$
\widetilde{J}_{1}=\int d^{D} l d^{D} k \frac{l_{0}^{p_{0}} k_{0}^{r_{0}}}{P_{1}^{t_{1}}(l+q) \widetilde{P}_{2}^{t_{2}}(l+k) \widetilde{P}_{3}^{t_{3}}(k)}
$$

where $t_{i} \in \mathbb{N}$ and $p_{0}$ and $r_{0}$ are non-negative integers. The integral $\widetilde{J}_{1}$ will be solved in the next section.

\footnotetext{
${ }^{3}$ If $w_{k}>0\left(\right.$ or $\left.w_{l}>0\right)$ we need only massive subtraction terms for the $l$ - (or the $k$-) integral.
} 
The second term of Eq. (21) is the tensor vacuum two-loop integral and has the form

$$
\begin{aligned}
& \int d^{D} l d^{D} k \frac{l_{0}^{p_{0}} k_{0}^{r_{0}}}{\left[l^{2}-m_{1}^{2}+i \rho\right]^{t_{1}}\left[(l+k)^{2}-m_{2}^{2}+i \rho\right]^{t_{2}}\left[k^{2}-m_{3}^{2}+i \rho\right]^{t_{3}}} \\
&=\int d^{D} l d^{D} k \frac{l^{\mu_{1}} \ldots l^{\mu_{p_{0}}} k^{\nu_{1}} \ldots k^{\nu_{r_{0}}}}{\left[l^{2}-m_{1}^{2}+i \rho\right]^{t_{1}}\left[(l+k)^{2}-m_{2}^{2}+i \rho\right]^{t_{2}}\left[k^{2}-m_{3}^{2}+i \rho\right]^{t_{3}}} \times \\
& \quad \times \frac{q_{\mu_{1}} \ldots q_{\mu_{p_{0}}} q_{\nu_{1}} \ldots q_{\nu_{r_{0}}}}{q^{r_{0}+p_{0}}} .
\end{aligned}
$$

An analytical solution of this integral with $t_{1}=t_{2}=t_{3}=1$ can be found in [12]. A closed analytical solution of a vacuum two-loop integral with arbitrary $t_{1}, t_{2}$ and $t_{3}$ can be obtained by integration by part and can be found in $[13,14]$. In this paper, we present another approach in calculating this integral. By using the decomposition in Lorentz covariant terms and then contracting with the external momenta $q_{\mu_{i}}$, the above integral is decomposed into massive vacuum integrals of the form

$$
\int d^{D} l d^{D} k \frac{\left(l^{2}\right)^{n}(l \cdot k)^{m}\left(k^{2}\right)^{p}}{\left[l^{2}-m_{1}^{2}+i \rho\right]^{t_{1}}\left[(l+k)^{2}-m_{2}^{2}+i \rho\right]^{t_{2}}\left[k^{2}-m_{3}^{2}+i \rho\right]^{t_{3}}}
$$

where $n, m, p$ are non-negative integer numbers. The integral is then reduced to products of one-loop integrals and (two-loop ) scalar massive vacuum integrals of the form

$$
\begin{aligned}
& S_{\left\{t_{1}, t_{2}, t_{3}\right\}}= \\
& \qquad \int d^{D} l d^{D} k \frac{1}{\left[l^{2}-m_{1}^{2}+i \rho\right]^{t_{1}}\left[(l+k)^{2}-m_{2}^{2}+i \rho\right]^{t_{2}}\left[k^{2}-m_{3}^{2}+i \rho\right]^{t_{3}}} .
\end{aligned}
$$

An efficient recursion relation to reduce Eq. (23) to Eq. (25) and the analytical solution of the massive vacuum integrals $S_{\left\{t_{1}, t_{2}, t_{3}\right\}}$ is presented in [11-13]

In the section II.5, we present another approach to solve the massive vacuum integral, which is more flexible from the computational point of view. But let us come back to the solution of the integral $\widetilde{J}_{1}$.

\section{II.4. The integral $\widetilde{J}_{1}$}

This section is dedicated to the analytical solution of the integrals $\widetilde{J}_{1}$ which appears in Eq. (22). We first write the integral more explicitly

$$
\widetilde{J}_{1}=\int d^{D} l d^{D} k \frac{l_{0}^{p_{0}} k_{0}^{r_{0}}}{P_{1}^{t_{1}}(l+q)\left[(l+k)^{2}\right]^{t_{2}}\left[k^{2}\right]^{t_{3}}}
$$


We can think of the integral over $k$ as a one-loop two-point integral with massless particles and "external" momentum $l$ with explicite sulution

$$
\begin{array}{rl}
\int d^{D} & k \frac{k_{0}^{r_{0}}}{\left[(l+k)^{2}\right]^{t_{3}}\left[k^{2}\right]^{t_{4}}}= \\
= & \frac{i(-1)^{D / 2}(\pi)^{D / 2}}{\Gamma\left(t_{3}\right) \Gamma\left(t_{4}\right)} \sum_{j=0}^{\left[r_{0}\right] / 2} \frac{r_{0} !\left(-l_{0}\right)^{r_{0}-2 j}\left(l^{2}\right)^{j+D / 2-t_{3}-t_{4}}}{\left(r_{0}-2 j\right) ! j ! 2^{2 j}} \times \\
& \times \Gamma\left(t_{4}+t_{4}-D / 2-j\right) \mathcal{B}\left(D / 2-t_{4}+r_{0}-j, D / 2-t_{3}+j\right)
\end{array}
$$

with $\left[r_{0}\right]=r_{0}$ if $r_{0}$ is even, and $\left[r_{0}\right]=r_{0}-1$ if $r_{0}$ is odd and $\mathcal{B}$ is the usual beta function defined by

$$
\mathcal{B}(x, y)=\frac{\Gamma(x) \Gamma(y)}{\Gamma(x+y)} .
$$

Substituting the right-hand side of Eq. (27) into Eq. (26), one ends up with threepoint one-loop integrals of the form

$$
\mathcal{K} \int d^{D} l \frac{l_{0}^{p_{0}^{\prime}}}{\left(l^{2}\right)^{\alpha} P_{1}^{t_{1}}(l+q)}
$$

where $\alpha$ is an integer when $D=4$, and $\mathcal{K}$ is a coefficient which depends on $D, t_{3}, t_{4}$ and $r_{0}$.

Because the $l$-integral is still UV-divergent, one can not set $D=4$, so $\alpha$ is in general a non-integer number. To calculate this kind of integral, we will use another approach rather than in [1] by employing the hypergeometric functions. Using the same reduction procedure as in the previous sections, the integral in Eq. (29) can be reduced to the form

$$
\mathfrak{L}=\int d^{D} l \frac{\left(l^{2}\right)^{n}}{\left[(l+q)^{2}-m_{1}^{2}+i \rho\right]^{t}\left[l^{2}\right]^{\alpha}}
$$

where $\alpha$ is a non-integer number. Now, using Feynman parametrisation and after shifting the momentum, one obtains

$$
\mathfrak{L}=\sum_{j_{1}=0}^{n} \sum_{j_{2}=0}^{j_{1}} \mathfrak{C} \int_{0}^{1} d x x^{a-1}(1-x)^{a^{\prime}-1}[(z-i \rho)-x]^{-b_{1}}
$$

with $j_{2}$ is even, $z=\frac{m^{2}}{q^{2}}, a=\alpha$ and

$$
a^{\prime}=\left(j_{1}+n-\alpha+\frac{D-j_{2}}{2}\right), \quad b_{1}=-\left(n-j_{1}-t-\alpha+\frac{D+j_{2}}{2}\right)
$$


and

$$
\begin{aligned}
\mathfrak{C} & =i\left(\begin{array}{l}
n \\
j_{1}
\end{array}\right)\left(\begin{array}{l}
j_{1} \\
j_{2}
\end{array}\right)(2)^{j_{2}} \pi^{D / 2}(-1)^{n-j_{1}+\frac{3 j_{2}}{2}-t-\alpha}\left(q^{2}\right)^{n-t-\alpha+D / 2} \\
& \times \frac{\mathcal{B}\left(t+\alpha-n+j_{1}-\frac{j_{2}+D}{2}, n-j_{1}+\frac{j_{2}+D}{2}\right)}{\mathcal{B}(t, \alpha)} \frac{\Gamma(D / 2) \Gamma\left(j_{2} / 2+1 / 2\right)}{\Gamma\left(D / 2+j_{2} / 2\right)} .
\end{aligned}
$$

With the help of a good mathematical handbook, the integral over $x$ in Eq. (31) can be integrated in terms of hypergeometric functions

$$
\mathfrak{L}=\sum_{j_{1}=0}^{n} \sum_{j_{2}=0}^{j_{1}} \mathfrak{C}(z-i \rho)^{-b_{1}} \mathcal{B}\left(a, a^{\prime}\right){ }_{2} F_{1}\left(a, b_{1}, a+a^{\prime}, \frac{1}{z-i \rho}\right) .
$$

For the scalar sunrise integral, this result is in agreement with [19]. For the general tensor case, an expansion method (at the pole $D=4$ ) for the hypergeometric functions ${ }_{2} F_{1}$ is presented in [20]. From the computational point of view, the expression Eq. (34) is ready to be evaluated with the help of nested sums as introduced in [20].

\section{II.5. The massive vacuum integral $S_{\left\{t_{1}, t_{2}, t_{3}\right\}}$}

To calculate the massive vacuum integral $S_{\left\{t_{1}, t_{2}, t_{3}\right\}}$, it is sufficient to consider the special case $t_{1}=t_{2}=t_{3}=1$. If $t_{1}>1$ ( or $t_{2}$ or $t_{3}>1$ ) then the integral can be evaluated as in a non-degenerate case. Let us consider the integral

$$
S_{\{1,1,1\}}=\int d^{D} l d^{D} k \frac{1}{P_{1} P_{2} P_{3}}
$$

with

$$
P_{1}=l^{2}-m_{1}^{2}+i \rho, \quad P_{2}=(l+k)^{2}-m_{2}^{2}+i \rho, \quad P_{3}=k^{2}-m_{3}^{2}+i \rho .
$$

By a simple transformation one obtains

$$
\begin{aligned}
S_{\{1,1,1\}}= & \int d^{D} l d^{D} k \frac{m_{1}^{2}}{P_{1} \widetilde{P}_{1} P_{2} P_{3}}+\int d^{D} l d^{D} k \frac{m_{2}^{2}}{\widetilde{P}_{1} P_{2} \widetilde{P}_{2} P_{3}} \\
& +\int d^{D} l d^{D} k \frac{1}{\widetilde{P}_{1} \widetilde{P}_{2} P_{3}}
\end{aligned}
$$

with

$$
\widetilde{P}_{1}=l^{2}, \quad \widetilde{P}_{2}=(l+k)^{2} .
$$

The first two terms of Eq. (35) are actually non-degenerate integrals which can be evaluated by the method in [1]. The last term which contains the overall divergence is now isolated in a simple integral $\widetilde{J}_{1}$ (see section II.4) which can be integrated analytically.

Having discussed the analytical solution of the divergent terms, in order to complete the calculation, one needs to find numerical solutions of the integrals which appear in the finite term of Eq. (21). The method of the calculation is presented in detail in [16]. In the next paragraphs, we will present a two-dimension representation of the finite part which can be evaluated numerically by computer programs such as VEGAS [21] or ParInt [22]. 
A two-dimension integral representation can be obtained by integrate over the variables $l_{\perp}, k_{\perp}$ and $z$ and finally results in the following form

$$
\begin{aligned}
\int d^{4} l \int d^{4} k \mathcal{J}_{2\left\{t_{i}\right\}}^{p_{0} r_{0}} L^{x_{l}} K^{x_{k}}= & \int_{-\infty}^{\infty} d l_{0} \int_{-\infty}^{\infty} d k_{0} \sum_{n_{1}=0}^{x_{k}} \sum_{n_{2}=0}^{x_{l}}\left(\begin{array}{c}
x_{k} \\
n_{1}
\end{array}\right)\left(\begin{array}{l}
x_{l} \\
n_{2}
\end{array}\right) \times \\
& \times(-1)^{n_{1}+n_{2}} l_{0}^{p_{0}} k_{0}^{r_{0}} \mathfrak{J}_{2}^{n_{1}, n_{2}}
\end{aligned}
$$

with

$$
\mathfrak{J}_{2}^{n_{1}, n_{2}}=8 \pi^{2} \int_{0}^{\infty} d l_{\perp} d k_{\perp} \int_{-1}^{1} d z l_{\perp}^{2} k_{\perp}^{2} \frac{\left(P_{1}^{t_{1}}\right)^{n_{2}-1}\left(P_{2}^{t_{2}} P_{3}^{t_{3}}\right)^{n_{1}-1}}{P_{1}^{t_{1}} P_{2}^{t_{2}} P_{3}^{t_{3}}\left(\bar{P}_{1}^{t_{1}}\right)^{n_{2}}\left(\widetilde{P}_{2}^{t_{2}} \widetilde{P}_{3}^{t_{3}}\right)^{n_{1}}} .
$$

Here, we write the integrals in terms of variables in the parallel and orthogonal space explicitly. The integrals $\mathfrak{J}_{2}$ are well defined. Further, $\mathfrak{J}_{2}$ can be decomposed into a sum of integrals of the form

$$
\widehat{J}_{2}=8 \pi^{2} \int_{0}^{\infty} d l_{\perp} d k_{\perp} \int_{-1}^{1} d z \frac{l_{\perp}^{2} k_{\perp}^{2}}{P_{1}^{t_{1}}(l+q) P_{2}^{t_{2}}(l+k) P_{3}^{t_{3}}(k)} .
$$

Integrating this integral by using the residuum theorem one obtains $[4,16]$.

$$
\widehat{J}_{2}=-4 \pi^{4} \ln \left(w_{1}+w_{2}+w_{3}\right)
$$

where

$$
\begin{aligned}
& w_{1}^{2}=\left(l_{0}+q_{0}\right)^{2}-m_{1}^{2}+i \rho, \\
& w_{2}^{2}=\left(l_{0}+k_{0}\right)^{2}-m_{2}^{2}+i \rho, \\
& w_{3}^{2}=k_{0}^{2}-m_{3}^{2}+i \rho .
\end{aligned}
$$

This result completes the method of integration for a degenerate tensor two-loop self-energy integral.

\section{SUN-RISE INTEGRAL AND NUMERICAL DISCUSSIONS}

The method of of integration for a degenerate tensor two-loop self-energy integral was implemented successfully in the program package XLOOPS-GiNaC. ${ }^{4}$ In this section, we present the results of calculating the sunrise integral ${ }^{5}$ using the program package XLOOPS-GiNaC. The results are then compared with the results of the existing results $[14,19,23,24]$.

The result of XLOOPS-GiNaC consists of two parts. One part is evaluated analytically in terms of the one-loop and/or hypergeometric functions. The other one is evaluated numerically by using Monte Carlo or Quasi-Monte Carlo methods with the help of the routines Vegas [21] or ParInt [22].

In the following, the sum of the analytical value and of the average of the result of the numerical integration will be called the main value. The error of Vegas (or ParInt)

\footnotetext{
${ }^{4}$ http: //wwwthep.physik.uni-mainz.de/ xloops

${ }^{5}$ Also called sunset integral
} 
will be called the error.

We first calculate the finite integral $T_{123 N}$ and compare our results with the results of [24]. The finite integral $T_{123 N}$ is defined by

$$
\begin{aligned}
T_{123 N}\left(p^{2} ; m_{1}^{2}, m_{2}^{2}, m_{3}^{2}\right): & =T_{123}\left(p^{2} ; m_{1}^{2}, m_{2}^{2}, m_{3}^{2}\right)-T_{123}\left(p^{2} ; m_{1}^{2}, 0, m_{3}^{2}\right) \\
& -T_{123}\left(p^{2} ; 0, m_{2}^{2}, m_{3}^{2}\right)+T_{123}\left(p^{2} ; 0,0, m_{3}^{2}\right)
\end{aligned}
$$

with

$$
\begin{aligned}
& T_{123}\left(p^{2} ; m_{1}^{2}, m_{2}^{2}, m_{3}^{2}\right)= \\
& \quad \int d^{D} l d^{D} k \frac{1}{\left[(l+p)^{2}-m_{1}^{2}+i \rho\right]\left[(l+k)^{2}-m_{2}^{2}+i \rho\right]\left[k^{2}-m_{3}^{2}+i \rho\right]} .
\end{aligned}
$$

In Tables 1 and 2 we show the results of XLOOPS-GiNaC and the results of [24] for small and large $p^{2}$ with different values for the masses. For the $p^{2}$-values above the threshold (large $p^{2}$ ), we also have a non-zero imaginary part, which is shown in the last two columns of Table 2. Each result in the table is calculated in less than one minute by XLOOPS$\mathrm{GiNaC}$ on a computer with Athlon 1.6 GHz CPU and 512 MB RAM. As seen in the tables, the differences are of order $10^{-3}$. The accuracy can be improved by increasing the number of iterations of Vegas and so the time of calculation also increases.

Table 1. Comparison for small $p^{2}$ of the real part of the subtracted sunrise in-

\begin{tabular}{|c|c|c|c|c|c|}
\hline$\overline{p^{2}}$ & $m_{1}$ & $m_{2}$ & $m_{3}$ & 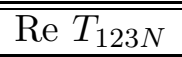 & Error \\
\hline 100 & 3 & 4 & 20 & $\begin{array}{l}-6.01715 \\
-6.02022\end{array}$ & \pm 0.00167 \\
\hline 150 & 3 & 4 & 20 & $\begin{array}{l}-6.39036 \\
-6.38877\end{array}$ & \pm 0.00017 \\
\hline 150 & 5 & 5 & 25 & $\begin{array}{l}-14.5339 \\
-14.5780\end{array}$ & \pm 0.00326 \\
\hline 200 & 5 & 5 & 25 & $\begin{array}{l}-15.0523 \\
-15.0542\end{array}$ & \pm 0.00192 \\
\hline
\end{tabular}
tegral $T_{123 N}$. In each box, in the first entry we show the value of Table 1 of [24] (small $p^{2}$ series expansion). The second entry is our result with its numerical error in the last column.

For a complete calculation of a sunrise integral, we compare the result of XLOOPS$\mathrm{GiNaC}$ with the result of $[14,23]$. We will follow the conventions of [14] where the sunrise integral is defined by

$$
\begin{aligned}
& F_{0}\left(D, m_{1}^{2}, m_{2}^{2}, m_{3}^{2}, p^{2}\right)= \\
& \quad \frac{\mu^{8-2 D}}{\left((2 \pi)^{D-2}\right)^{2}} \int d^{D} k_{1} \int d^{D} k_{2} \frac{1}{\left(k_{1}^{2}+m_{1}^{2}\right)\left(k_{2}^{2}+m_{2}^{2}\right)\left(\left(p-k_{1}-k_{2}\right)^{2}+m_{3}^{2}\right)}
\end{aligned}
$$


Table 2. Comparison for large $p^{2}$ of the real and imaginary part of the subtracted sunrise integral $T_{123 N}$. In each box, in the first entry we show the value of Table 2 of [24] (large $p^{2}$ series expansion). The second entry is our result with its numerical error in the next column.

\begin{tabular}{||l|l|l|l||r|l||l|l||}
\hline \hline$p^{2}$ & $m_{1}$ & $m_{2}$ & $m_{3}$ & Re $T_{123 N}$ & Vegas Error & $\operatorname{Im} T_{123 N}$ & Vegas Error \\
\hline 80 & 2 & 3 & 2 & 0.58743 & & -11.2628 & \\
& & & & 0.58585 & \pm 0.00048 & -11.2632 & \pm 0.0002 \\
\hline 100 & 2 & 3 & 4 & -0.32864 & & -11.8459 & \\
& & & & -0.32028 & \pm 0.00118 & -11.8460 & \pm 0.0002 \\
\hline 150 & 3 & 4 & 4 & -1.26795 & & -26.4912 & \\
& & & & -1.21248 & \pm 0.00140 & -26.4912 & \pm 0.0005 \\
\hline 200 & 2 & 3 & 4 & 1.69608 & & -6.02417 & \\
& & & & 1.69549 & \pm 0.00022 & -6.02412 & \pm 0.0001 \\
\hline 250 & 4 & 4 & 4 & 2.64395 & & -27.6090 & \\
& & & & 2.65248 & \pm 0.00893 & -27.6098 & \pm 0.0006 \\
\hline \hline
\end{tabular}

where

$$
\mu=m_{1}+m_{2}+m_{3}
$$

The integral $F_{j}\left(D, m_{1}^{2}, m_{2}^{2}, m_{3}^{2}, p^{2}\right)$ can be parametrized by

$$
\begin{aligned}
& F_{0}\left(D, m_{1}^{2}, m_{2}^{2}, m_{3}^{2}, p^{2}\right)= \\
& C^{2}(D)\left\{\frac{1}{(D-4)^{2}} F_{0}^{(-2)}+\frac{1}{(D-4)} F_{0}^{(-1)}+F_{0}^{(0)}\right\}
\end{aligned}
$$

with

$$
C(D)=(2 \sqrt{\pi})^{4-D} \Gamma(3-D / 2) .
$$

For the divergent terms we obtain

$$
\begin{aligned}
& F_{0}^{(-2)}=-\frac{1}{8} \sum_{i=1}^{3} m_{i}^{2} \\
& F_{0}^{(-1)}=\frac{1}{8}\left\{-\frac{p^{2}}{4}+\frac{3}{2} \sum_{i=1}^{3} m_{i}^{2}-\sum_{i=1}^{3} m_{i}^{2} \ln \left(\frac{m_{i}^{2}}{\mu^{2}}\right)\right\}
\end{aligned}
$$

which is in agreement with the results of $[14,23]$. Note that our result differs with the one of [14] by a minus sign in the term $\frac{p^{2}}{4}$ of $F_{0}^{(-1)}$. This is due to the fact that in [14] the integral is evaluated in Euclidian space. 
Table 3. Comparison for the sunrise integral. The masses (in $\mathrm{GeV}$ ) are $m_{1}=1$, $m_{2}=9, m_{3}=200$ and $\mu=m_{1}+m_{2}+m_{3}$. Results in columns A are reproduced from [14]. The results of XLOOPS-GiNaC and their errors are shown in column $\mathrm{B}$ and $\mathrm{C}$, respectively.

\begin{tabular}{||l|l|l|l||}
\hline \hline$p_{r}^{2}$ & $\mathrm{~A}$ & $\mathrm{~B}$ & $\mathrm{C}$ \\
\hline-1 & -0.279454902855371 & -0.279819 & $\pm 6.6 \mathrm{E}-4$ \\
\hline-0.99 & $-0.2798928396415(5)$ & -0.280351 & $\pm 0.9 \mathrm{E}-4$ \\
\hline$p_{p s 3, r}^{2}$ & -0.280281633048667 & -0.280442 & $\pm 4.4 \mathrm{E}-4$ \\
\hline-0.9 & $-0.2836780811878(5)$ & -0.283169 & $\pm 5.6 \mathrm{E}-4$ \\
\hline$p_{p s 2, r}^{2}$ & -0.286233415451605 & -0.285265 & $\pm 16.3 \mathrm{E}-4$ \\
\hline-0.825 & $-0.2866587055221(5)$ & -0.281268 & $\pm 4.2 \mathrm{E}-4$ \\
\hline$p_{p s 1, r}^{2}$ & -0.286906928933491 & -0.286141 & $\pm 5.7 \mathrm{E}-4$ \\
\hline-0.8 & $-0.2876221116285(5)$ & -0.288281 & $\pm 5.4 \mathrm{E}-4$ \\
\hline-0.1 & $-0.3101507246241(3)$ & -0.310516 & $\pm 1.9 \mathrm{E}-4$ \\
\hline 0 & -0.312816604092084 & -0.312800 & $\pm 0.1 \mathrm{E}-4$ \\
\hline \hline
\end{tabular}

In table 3 we present the numerical values of $\frac{F_{0}^{(0)}}{\mu^{2}}$ as a function of $\frac{p^{2}}{\mu^{2}}$. The abbreviations which are used in the table are

$$
\begin{aligned}
& p_{p s 1, r}^{2}=\frac{\left(m_{1}-m_{2}-m_{3}\right)^{2}}{\mu^{2}}, \\
& p_{p s 2, r}^{2}=\frac{\left(m_{1}-m_{2}+m_{3}\right)^{2}}{\mu^{2}}, \\
& p_{p s 3, r}^{2}=\frac{\left(m_{1}+m_{2}-m_{3}\right)^{2}}{\mu^{2}} .
\end{aligned}
$$

In column A we show the results of [14] using differential equation techniques. The results of XLOOPS-GiNaC and their errors are shown in columns B and C. The difference of the results is of order $10^{-3}$ where we use $10^{5} \times 10$ iterations per integration for Vegas.

\section{CONCLUSIONS}

We have shown that with an extension of the method in [1], a subset of degenerate two-loop self-energy integral can be reduced to finite integral representations and UV-divergent analytic expressions in a similar manner as in non-degenerate cases. The complete method provides a general algorithm of evaluating an arbitrary two-loop selfenergy integral which can be easily implemented into a computer program.

It is worth to note that to obtain a stable numerical result, our method needs not to consider different kinematic regions of the integrals such as below or above the threshold or around the pseudo-thresholds.

Even though the finite part of integrals is evaluated numerically in terms of twodimensions integrals, we are still able to obtain a good accuracy in a reasonable computation time. 


\section{ACKNOWLEDGEMENTS}

Do Hoang Son (H.S. Do) was supported by the National Fundamental Research program of the Ministry of Science and Technology of Vietnam; The Graduiertenkolleg "Eichtheorien - experimentelle Tests und theoretische Grundlagen" in the University Mainz and in part by a grant of the BMBF. He would like to thank H. Spiesberger, S. Groote and M. Knodel for fruitful discussion; C. Bauer, A. Frink, R. Kreckel and J. Vollinga for the excellent computer library GiNaC.

\section{REFERENCES}

[1] D. Kreimer, Mod. Phys. Lett. A9 (1994) 1105.

[2] S. Actis, A. Ferroglia, G. Passarino, M. Passera, and S. Uccirati, Nucl.Phys. B 703 (2004) 3.

[3] J. R. Andersen et al., The SM and NLO Multileg Working Group: Summary Report, (2010).

[4] H.S. Do, PhD thesis, Johannes Gutenberg Universität Mainz, 2003.

[5] J. Franzkowski, PhD thesis, Johannes Gutenberg Universität Mainz, 1997.

[6] C. Bauer and H. S. Do,Comput. Phys. Commun. 144 (2002) 154, [hep-ph/0102231].

[7] S. Groote and M. Knodel, Eur. Phys. J. C46 (2006) 157-178.

[8] R. Scharf, PhD thesis, Universität Wurzburg, 1994.

[9] G. Passarino and S. Uccirati, Nucl. Phys. B629 (2002) 97.

[10] G. Weiglein, R. Scharf, and M. Băohm, Nucl. Phys. B416 (1994) 606.

[11] A. I. Davydychev and J.B. Tausk, Nucl. Phys. B397 (1993) 123.

[12] A. I. Davydychev, V. A. Smirnov, and J.B. Tausk, Nucl. Phys. B 410 (1993) 325, [hep- ph/9307371].

[13] K. G. Chetyrkin, A Closed Analytical Formula for Two-loop Massive Tadpoles with Arbitrary Tensor Numerators, World Scientific, Singapore, 1994.

[14] M. Caffo, H. Czyż, and Remiddi, Nucl.Phys. B634 (2002) 309, [hep-ph/0203256].

[15] L. Brucher, J. Franzkowski, A. Frink, and D. Kreimer, Acta Phys. Polon. B28 (1997) 835- 840.

[16] D. Kreimer, PhD thesis, Johannes Gutenberg Universitát Mainz, 1992.

[17] J. Collins, Renormalization, Cambridge Univ. Press, 1984.

[18] Ghinculov and York-Peng Yao, Nucl. Phys. B516 (1998) 385, [hep-ph/9702266].

[19] S. Bauberger, F. A. Berends, M. Băohm, and M. Buza, Nucl. Phys. B434 (1995) 383,

[20] S. Weinzierl, Comp. Phys. Comm. 145 (2002) 357, [math-ph/0201011].

[21] G. P. Lepage, J. Comp. Phys. 27 (1978) 192

[22] L. Cucos and E. de Doncker, Proceedings of the High Performance Computing Symposium 2002 (HPC'02), (2002)

[23] S. Groote and A.A. Pivovarov, Nucl. Phys. B580 (2000) 459.

[24] F.A. Berends, M. Bohm, M. Buza, and R. Scharf, Z. Phys. C63 (1994) 227.

Received 26 October 2009. 